ARTICLE

\title{
Kinetic Monte Carlo Annealing Simulation of Cascade Damage in $\alpha$-Fe
}

\author{
Tomoaki SUZUDO ${ }^{1, *}$, Stanislav I. GOLUBOV ${ }^{2,3}$, Roger E. STOLLER ${ }^{2}$, \\ Masatake YAMAGUCHI ${ }^{1}$, Tomohito TSURU $^{1}$ and Hideo KABURAKI ${ }^{1}$ \\ ${ }^{1}$ Japan Atomic Energy Agency, 2-4 Shirane Shirakata Tokai-mura, 319-1195 Japan \\ ${ }^{2}$ Materials Science and Technology Division Oak Ridge National Laboratory, Oak Ridge, USA \\ ${ }^{3}$ Department of Materials Science and Engineering University of Tennessee, Knoxville, TN USA
}

\begin{abstract}
Molecular dynamics is a useful tool for simulating cascade damage in metals and alloys, but the time scale accessible to molecular dynamics is only about $10^{-10} \mathrm{~s}$. Kinetic Monte Carlo can be used to simulate annealing of cascade damage to permit analysis of the longer time evolution of cascade damage. We conducted a series of such annealing simulations in $\alpha$-Fe. The number of surviving displacements before annealing is $\sim 0.3$ of the Norgett-Robinson-Torrens (NRT) value in the case of primary knock-on atoms with energy more than $\sim 10 \mathrm{keV}$, and it decreased by $\sim 30 \%$ during the annealing at $300 \mathrm{~K}$ because of recombination of vacancies and self-interstitial atoms. The recombination ratio increased as the annealing temperature increased. These results can be meaningfully applied in models such as mean field reaction rate theory used to simulate long-term radiation damage accumulation. We also demonstrated that 1D motion of small SIA clusters can substantially influence the long-term accumulation of cascade damage.
\end{abstract}

KEYWORDS: cascade damage, kinetic Monte Carlo, annealing simulation, $\alpha-F e$, damage production efficiency, defect clusters

\section{Introduction}

Irradiation damage modeling of metals and alloys is of great importance, not only for ensuring the safety of current light-water-cooled nuclear reactors but also for designing future Generation IV and fusion reactors. In spite of the substantial level of research carried out for a few decades, we do not yet have models that can accurately predict the lifetime of structural materials exposed to a long period of neutron irradiation.

One of the difficulties of such modeling is the complexity of cascade damage production, which involves many atomic displacements produced by primary knock-on atoms (PKAs). Some cascade damage directly produces defect clusters, such as vacancy clusters and self-interstitial atom (SIA) clusters; these clusters provide nuclei for larger defects which can give rise to dimensional and mechanical property changes. Molecular dynamics with a proper interatomic potential is the only theoretical tool that can model such cascade damage, and it is extensively applied for this purpose. ${ }^{1-3)}$

Although computer technology development has dramatically improved the computational efficiency of molecular dynamics simulations, the number of atoms and the temporal length molecular dynamics can deal with are typically $\sim 10^{7}$ atoms and $\sim 10^{-10} \mathrm{~s}$, respectively. It is consequently obvious that the usage of molecular dynamics (MD) is limited for practical prediction of microstructural evolution.

A possible solution is to apply the kinetic Monte Carlo

*Corresponding author, E-mail:suzudo.tomoaki@jaea.go.jp

(C) 2011 Atomic Energy Society of Japan, All Rights Reserved.
(kMC) method in addition to MD. The time scale accessible to kMC is much longer than that of MD. Thus, it is possible to track the time development of a single cascade until the spatial correlations between defects almost disappear, so that the results can be applied to the radiation model in mean field rate theory (MFRT) $)^{4}$ simulations.

There are a several previous studies on this topic, involving annealing of $\mathrm{Cu}^{5)}$ and $\alpha-\mathrm{Fe}^{6-9)}$ cascades. To date, there has been no annealing study of Fe that includes damage energies more than $40 \mathrm{keV}$, although such cases are important for the study of nuclear fusion application. In addition, the understanding of the motion of self-interstitial atoms (SIAs) and SIA clusters has been significantly improved by ab initio calculations. ${ }^{10,11)}$ It is thus worth revisiting the annealing of irradiation damage in $\alpha$-Fe using cascade data for recoil energies of more than $40 \mathrm{keV}$ and updated defect migration parameters.

The primary goal of this paper is to supply statistically reliable information on how various cascades in $\alpha$-Fe evolve at constant temperature by adding information from cascades with higher recoil energy. For this purpose, we utilized a comprehensive database of cascade damage of $\alpha$-Fe for a range of PKA energies. ${ }^{2)}$ We especially focused on the development of defect clusters and surviving displacements.

\section{Numerical Method}

\section{Pre-Processing of Cascade Damage Data}

As mentioned above, a cascade damage database was utilized in the present study. To conduct the cascade simulations, a block of atoms is thermally equilibrated at 
Table 1 Cascade data sets used in the present annealing study

\begin{tabular}{ccccc}
\hline$E_{\mathrm{PKA}}[\mathrm{keV}]^{*}$ & $E_{\mathrm{D}}[\mathrm{keV}]$ & $\begin{array}{c}\text { Number of } \\
\text { cascades }\end{array}$ & $\begin{array}{c}\text { NRT } \\
\text { displacements }\end{array}$ & $\begin{array}{c}\text { Mean number of } \\
\text { displacements }\end{array}$ \\
\hline 0.1158 & 0.1 & 40 & 1 & 1.45 \\
0.2357 & 0.2 & 21 & 2 & 2.05 \\
0.6046 & 0.5 & 20 & 5 & 3.80 \\
1.237 & 1.0 & 12 & 10 & 6.08 \\
2.538 & 2.0 & 10 & 20 & 10.0 \\
6.605 & 5.0 & 9 & 50 & 22.0 \\
13.71 & 10 & 15 & 100 & 34.0 \\
28.76 & 20 & 10 & 200 & 61.0 \\
44.67 & 16 & 300 & 95.3 \\
61.32 & 30 & 400 & 128 \\
78.69 & 40 & 9 & 500 & 170 \\
175.8 & 50 & 10 & 1000 & 331 \\
425.5 & 100 & 9 & 2000 & 683 \\
\hline
\end{tabular}

*For discussion of PKA and damage energy, see Ref. 1).

**The threshold energy of displacement of $0.04 \mathrm{keV}$ was used.

100 K. Different equilibrated atom blocks give different time-developments of cascade damage, and, in this way, it is possible to increase the statistical reliability of the results. The simulations are initiated by giving a lattice atom a specified amount of kinetic energy, which is analogous to the damage energy $E_{\mathrm{D}}$ in the Norgett-Robinson-Torrens (NRT) model. ${ }^{12)}$ The cascade simulations last until the phase of fast in-cascade recombination of vacancies and SIAs is completed and the atom block returns to near thermal equilibrium. A detailed description of this database is given in Ref. 2), and the part of the database utilized in the present study is summarized in Table 1. As seen in the table, at least nine unique individual cascades are available for each damage energy.

We are interested in surviving defects such as vacancies and SIAs. By comparing the final snapshot of the atomic block with the ideal crystal, all the surviving vacancies and SIAs are recognized. Through this data processing, we determined the total number of displacements and their configuration for each cascade case.

Some of the vacancies and SIAs form clusters directly during the cascade event. Vacancy clusters were identified on the basis of the binding energy of a pair of vacancies. For this purpose, we conducted $a b$ initio and molecular statics simulations, as seen in Table 2. The detailed methodology used for the $a b$ initio studies is given in Ref. 13). For the molecular statics studies, the conjugate gradient method was used for relaxation of atomic configuration, where the interatomic potential proposed in Ref. 14) was used. It is obvious that only the first and second nearest neighbor (NN) pairs have a meaningful value of binding energy, and these pairs were used as the basis for defining vacancy clusters.

For SIA clusters, using binding-energy as a basis to determine clusters becomes much more cumbersome, because there are many types of SIAs, such as
Table 2 Binding energy for variously-configured pair of vacancies

\begin{tabular}{cccc}
\hline $\begin{array}{c}\text { Type } \\
\text { of } \\
\mathrm{NN}^{*}\end{array}$ & Distance $(\AA)$ & $\begin{array}{c}\text { Binding } \\
\text { energy } \\
(\mathrm{ab} \text { initio) } \\
{[\mathrm{eV}]}\end{array}$ & $\begin{array}{c}\text { Binding } \\
\text { energy } \\
\text { (Molecula } \\
\text { r statics) } \\
{[\mathrm{eV}]}\end{array}$ \\
\hline 1st NN & 2.45 & -0.13 & -0.143 \\
2nd NN & 2.83 & -0.21 & -0.190 \\
3rd NN & 4.00 & 0.016 & 0.065 \\
4th NN & 4.69 & -0.042 & -0.042 \\
5th NN & 4.90 & -0.040 & 0.023 \\
\hline
\end{tabular}

*”NN" stands for nearest neighbor.

$<110>$-dumbbell and <111>-crowdion. Besides, each type of dumbbell and crowdion has a variety of equivalent directions. Thus in the present study SIA clusters were recognized simply based on the distance between two SIAs. As adopted in Ref. 15), two SIAs closer than $1.5 a_{0}$ were determined to be part of an SIA cluster, where $a_{0}=2.83 \AA$ is the lattice constant of $\alpha$-Fe. This criterion practically indicates that first, second and third NN dumbbell pairs were designated as part of an SIA cluster regardless of their configuration.

\section{Annealing Method}

We adopted an object kinetic Monte-Carlo (OKMC) method $^{16,17)}$ for the annealing simulations of cascade damage. This method is capable of tracking the fate of various types of defects in materials. In the framework of OKMC, vacancies, SIAs and their clusters are treated as "objects" that migrate and interact with others. The event-based time-development algorithm is adopted in the method, and the following steps are iterated: (a) occurrence probabilities of all the considered events are calculated on the basis of 
present defect configuration; (b) an event is selected randomly where the selection probability is proportional to the occurrence probability of each event; and (c) the time advances by $\delta$;

$$
\delta t=-\left(\log r / k_{t o t}\right)
$$

where $k_{\text {tot }}$ is the sum of probabilities of all the events; $r$ a uniform random number in the range between zero and one. A good introduction to the kinetic Monte-Carlo method is given in Ref. 18).

We adopted a cubic simulation box of $\alpha$-Fe; the size of the box is $(100 \mathrm{~nm})^{3}$ and each axis is parallel to a $<100>$-direction. Initial defect distributions obtained from the MD simulations were placed in the center of the box. A free boundary condition was adopted in all the three directions, such that any defects which reached one of the boundaries were removed from the simulation box, and such defects were counted as escaped defects.

Defect types considered in this study are vacancy, SIA, vacancy cluster, and SIA cluster, and for convenience sake they are denoted by $V_{1} I_{1}, V_{n}$, and $I_{n}$, respectively in the following, where $n$ is the number of vacancies or SIAs in the cluster. As experimentally observed, we assumed that each SIA has the form of a dumbbell or crowdion, and that vacancy clusters are spherical objects. SIA clusters in reality form loops, but we assumed that these were spherical because this approximation is valid when clusters are small. ${ }^{19)}$

We assumed that all the defects are mobile except vacancy clusters because migration energy of vacancy clusters is much larger than that of other types of defects and the migration of vacancy clusters can be ignored. The probability of defect migration $v_{\mathrm{m}}$ is given by

$$
v_{\mathrm{m}}=v_{\mathrm{m}}{ }^{0} \exp \left\{-E_{\mathrm{m}} / k T\right\},
$$

where $v_{\mathrm{m}}{ }^{0}$ is the pre-exponential factor for $v_{\mathrm{m}}$; $E_{\mathrm{m}}$ the migration energy; $k$ the Boltzmann constant; $T$ the absolute temperature. The value of $v_{\mathrm{m}}{ }^{0}$ is given by $d D_{0} / \delta^{2}$, where $d$ is the dimensionality of migration; $D_{0}$ the pre-exponential factor of diffusion constant; $\delta$ the jump distance in migration ( $\sim 0.249 \mathrm{~nm}$ for $\alpha$-Fe crystal). In 3D-motion mode, defects can jump to one of the eight nearest neighboring positions ( $\pm a_{0} / 2, \pm a_{0} / 2, \pm a_{0} / 2$ ). In $1 \mathrm{D}$-motion mode, on the other hands, jumping only to two neighboring positions in a specified direction is allowed. Discussion of the dimensionality of $\mathrm{I}_{\mathrm{n}}$ 's motion of $\alpha$-Fe can be found elsewhere. ${ }^{11,20-22)}$ Taken together, those studies indicate: $\mathrm{I}_{1}$ and $I_{2}$ have $3 D$ motion; $I_{3}, I_{4}$ and $I_{5}$ have mixed $1 D / 3 D$ motion; $I_{n}(n \geq 6)$ has $1 D$ motion. In the present study, defects reactions occur only in cascades and ignored 3D motion for $\mathrm{I}_{3}, \mathrm{I}_{4}$ and $\mathrm{I}_{5}$. Because a recent molecular dynamics study ${ }^{21)}$ claims that $\mathrm{I}_{3}$ has almost 3D motion, we check the influence of this parameter later in Section III. 3. We determined $E_{\mathrm{m}}$ for $I_{n}(n \geq 3)$ based on a recent thorough molecular dynamics study by Anento et al. ${ }^{22)} D_{0}$ for each migrating defect can be roughly estimated from molecular dynamics, and $D_{0}$ for $\mathrm{I}_{\mathrm{n}}$ were determined with reference to the results given in
Table 3 Parameters related to defect migration used in the OKMC model

\begin{tabular}{ccrc}
\hline Defect type & $d$ & $E_{m}[\mathrm{eV}]$ & $D_{0}\left[\mathrm{~m}^{2} / \mathrm{s}\right]$ \\
\hline $\mathrm{V}_{1}$ & 3 & 0.65 & $1.0 \times 10^{-6}$ \\
$\mathrm{I}_{1}$ & 3 & 0.30 & $1.0 \times 10^{-6}$ \\
$\mathrm{I}_{2}$ & 3 & 0.42 & $1.0 \times 10^{-5}$ \\
$\mathrm{I}_{3}$ & $1^{*}$ & 0.15 & $1.0 \times 10^{-7}$ \\
$\mathrm{I}_{4}$ & 1 & 0.15 & $1.0 \times 10^{-7}$ \\
$\mathrm{I}_{\mathrm{n}}(\mathrm{n} \geq 5)$ & 1 & 0.04 & $1.0 \times 10^{-7}$ \\
\hline
\end{tabular}

*Only in Section III. $3, d=3$ for $\mathrm{I}_{3}$ is discussed.

Refs. 21, 22). The values for $D_{0}, E_{\mathrm{m}}$, and $d$ for each defect type are given in Table 3.

Another event to consider is vacancy dissociation from a vacancy cluster. The probability of the dissociation $v_{\mathrm{d}}$ is given by

$$
v_{\mathrm{d}}=v_{\mathrm{m}}{ }^{0} \exp \left\{-\left(E_{\mathrm{m}}+E_{\mathrm{d}}\right) / k T\right\},
$$

where $E_{\mathrm{d}}$ is the binding energy of vacancy to vacancy cluster. The general expression for the binding energy takes the following forms:

$$
E_{d}=\left(E_{2 V}-E_{f}\right) \frac{n^{2 / 3}-(n-1)^{2 / 3}}{2^{2 / 3}-1}
$$

where $n$ is the number of vacancies in the cluster before dissociation; $E_{2 \mathrm{~V}}$ the di-vacancy binding energy; $E_{\mathrm{f}}$ the vacancy formation energy. We used $1.6 \mathrm{eV}$ and $0.2 \mathrm{eV}$ for $E_{2 \mathrm{~V}}, E_{\mathrm{f}}$, respectively. We ignored SIA dissociation from SIA clusters as these events are much rarer than other events.

Each migrating defect can react with another nearby defects. For instance, recombination of Frenkel pair:

$$
\mathrm{V}_{1}+\mathrm{I}_{1} \rightarrow 0,
$$

and reactions between a vacancy (or an SIA) and a defect cluster such as

$$
\begin{array}{ll}
\mathrm{V}_{1}+\mathrm{V}_{\mathrm{n}} \rightarrow \mathrm{V}_{\mathrm{n}+1}, & \mathrm{~V}_{1}+\mathrm{I}_{\mathrm{n}} \rightarrow \mathrm{I}_{\mathrm{n}-1} \\
\mathrm{I}_{1}+\mathrm{V}_{\mathrm{n}} \rightarrow \mathrm{V}_{\mathrm{n}-1}, & \mathrm{I}_{1}+\mathrm{I}_{\mathrm{n}} \rightarrow \mathrm{I}_{\mathrm{n}+1}
\end{array}
$$

were considered. The probability of reactions between clusters is small, because clusters produced by cascade are not many. We thus ignored interactions between clusters. The reactions described in Eqs. (5), (6) and (7) spontaneously occur when the distance between the defects is less than the reaction distance $d_{\mathrm{r}}$,

$$
d_{\mathrm{r}}=r_{i}+r_{j}+\Delta d,
$$

where $r_{i}$ and $r_{j}$ are reaction radii of the two reacting defects $i$ and $j ; \Delta d$ the distance for spontaneous reaction. The reaction radius of a defect is based on the assumption that clusters are spherical, i.e.

$$
r_{i}=\left(3 n_{i} V_{\mathrm{at}} / 4 \pi\right)^{1 / 3},
$$

where $n_{i}$ is the number of vacancies/SIAs contained in the defect $i$ and $V_{\text {at }}$ is iron's atomic volume $\left(\sim 1.18 \times 10^{-27} \mathrm{~m}^{3}\right)$. We used $\Delta d=0.164 \mathrm{~nm}$. This value makes any first, second and third NN defect pairs spontaneously react. 


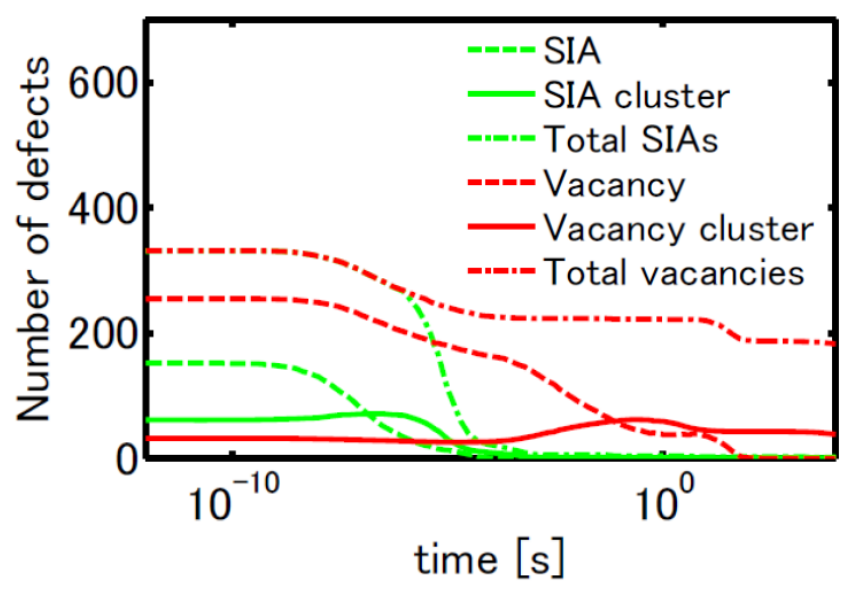

Fig. 1 Mean number of surviving defects for $E_{\mathrm{D}}=100 \mathrm{keV}$

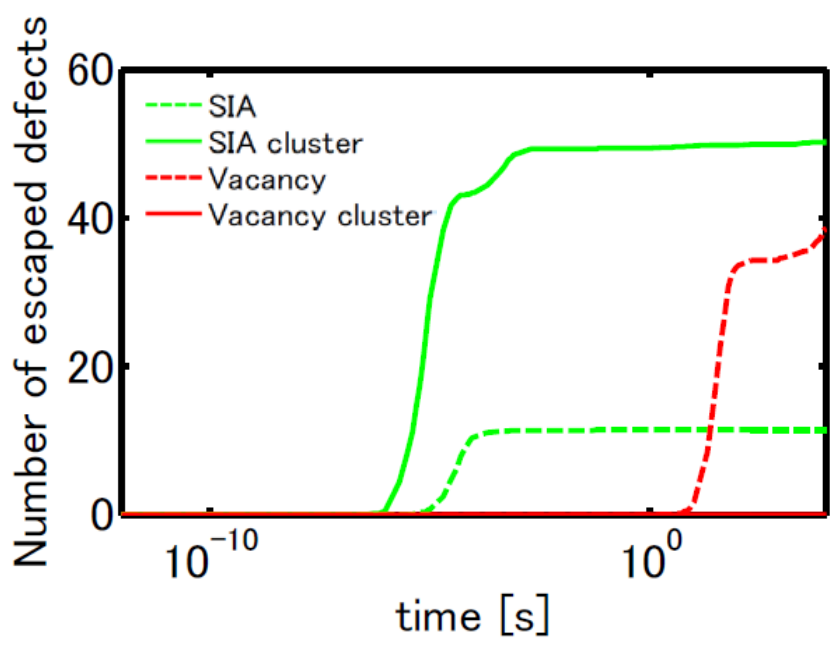

Fig. 2 Mean number of escaped defects for $E_{\mathrm{D}}=100 \mathrm{keV}$

\section{Results and Discussion}

\section{Annealing Simulations at $300 \mathrm{~K}$}

In the case of pure iron, cascade damage produced by molecular dynamics simulation is almost independent of irradiation temperature up to $900 \mathrm{~K},{ }^{2)}$ and the data set shown in Table 1 can be used for annealing simulations at different temperatures. We conducted annealing simulations at $300 \mathrm{~K}$ for $10^{4} \mathrm{~s}$.

At first, we will have a look at the case for $E_{\mathrm{D}}=100 \mathrm{keV}$, which is one of the larger damage energies in the present study. Figure 1 shows the time evolution of the number of residual defects in the simulation box. Note that these graphs show the values averaged over different cascade damage data sets with the same damage energy. The earliest change in the graphs is seen at $\sim 10^{-8} \mathrm{~s}$.

This is caused by the migration of single SIAs and leads to the formation of new SIA clusters and recombination with vacancies. Figure 2 shows the time evolution of the number of defects escaping from the simulation box; these are also mean values. This figure indicates that no defects escape during the first $\sim 10^{-6} \mathrm{~s}$. Following this, some SIA clusters begin to escape due to rapid one-dimensional migration. From Fig. 2, we can recognize that SIA clusters migrating (a)

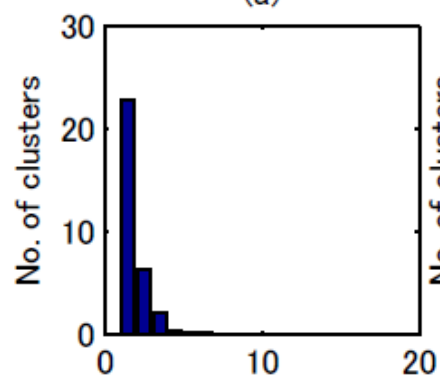

No. of point defects (b)

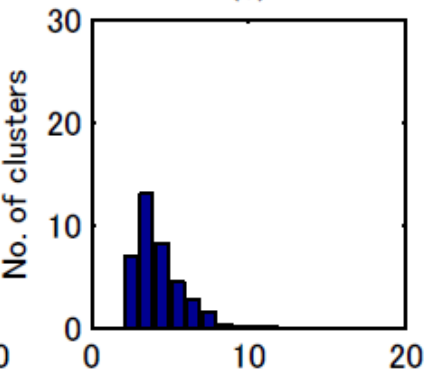

No. of point defects

Fig. 3 Vacancy cluster size distribution for $\mathrm{ED}=100 \mathrm{keV}$ before annealing (a) and after annealing (b)

(a)

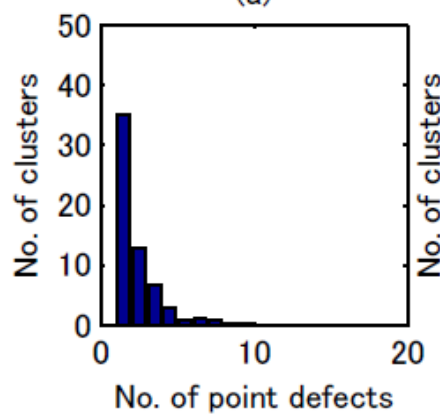

(b)

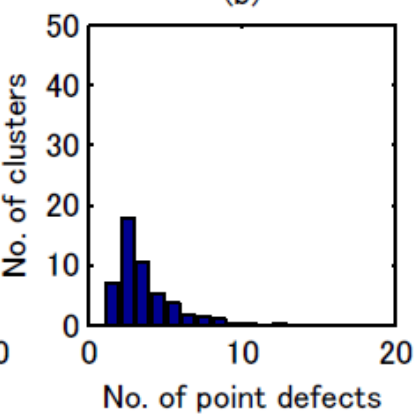

Fig. 4 SIA cluster size distribution for $E_{\mathrm{D}}=100 \mathrm{keV}$ before annealing (a) and escaped during annealing (b)

one-dimensionally immediately escape from the simulation box. At $\sim 10^{-5} \mathrm{~s}$ single SIAs also reach the boundary and escape, and SIA clusters migrating three-dimensionally escape at $\sim 10^{-4} \mathrm{~s}$; this is responsible for the second period of growth in the number of escaped SIA clusters in Fig. 2. In the meantime, single vacancies start migrating and forming clusters. This change is recognized as a decrease in vacancies and an increase in vacancy clusters. Vacancies escape from the simulation box at $\sim 10 \mathrm{~s}$. Finally at $\sim 10^{3} \mathrm{~s}$ some vacancies start dissociating from the clusters and escape from the box.

Figure $\mathbf{3}$ is the mean size distribution of vacancy clusters before and after annealing for this $E_{\mathrm{D}}$. Before annealing, i.e. just after the cascade event, most clusters are composed either of two or three vacancies. As recognized by the comparison between Figs. 3(a) and (b), the surviving vacancy clusters after annealing were generally larger than those directly created by cascade damage. In particular, none of the smallest cluster $V_{2}$ were left, because they all became larger or dissociated into two individual vacancies.

Figure 4(a) is the mean size distribution of initial SIA clusters for $E_{\mathrm{D}}=100 \mathrm{keV}$. Similar to vacancy clusters, most of the SIA clusters were composed of two or three SIAs and $I_{n} S \quad(n \geq 5)$ were rare. Figure 4(b) is the mean size distribution of SIA clusters that escaped for the same case.

The shape of this histogram is similar to that before annealing except that the number of smallest clusters $\left(\mathrm{I}_{2}\right)$ is less than that of $\mathrm{I}_{3}$. This is attributed to the fact that $\mathrm{I}_{2}$ with 3D motion has a larger chance to grow or to shrink by interaction than $\mathrm{I}_{\mathrm{n}}(n>2)$ with 1D motion. 


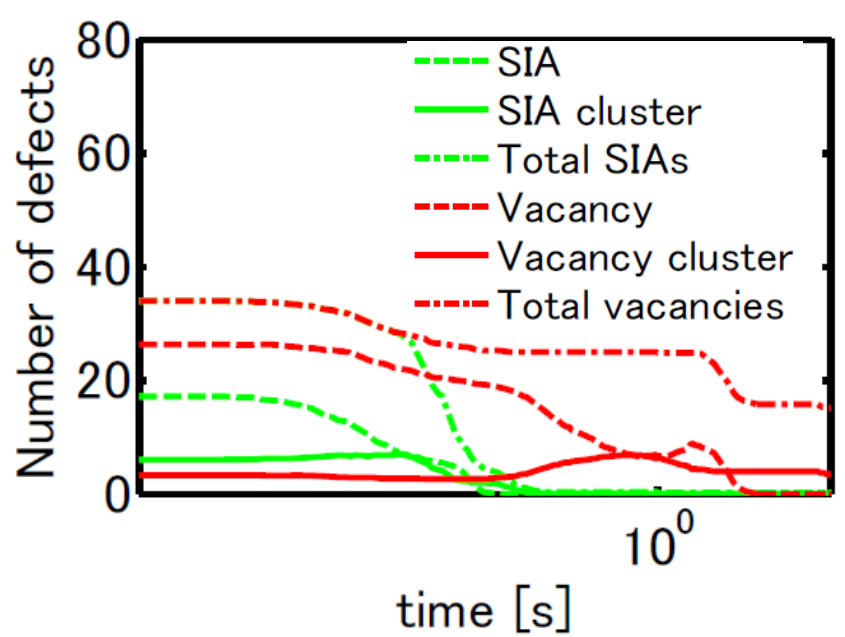

Fig. 5 Mean number of surviving defects for $E_{\mathrm{D}}=10 \mathrm{keV}$

(a)

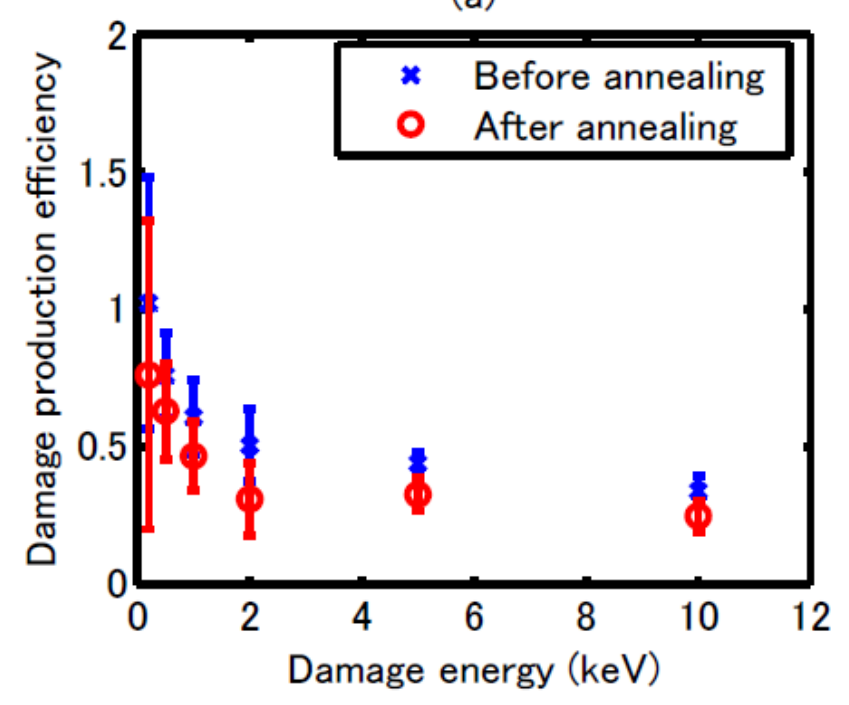

(b)

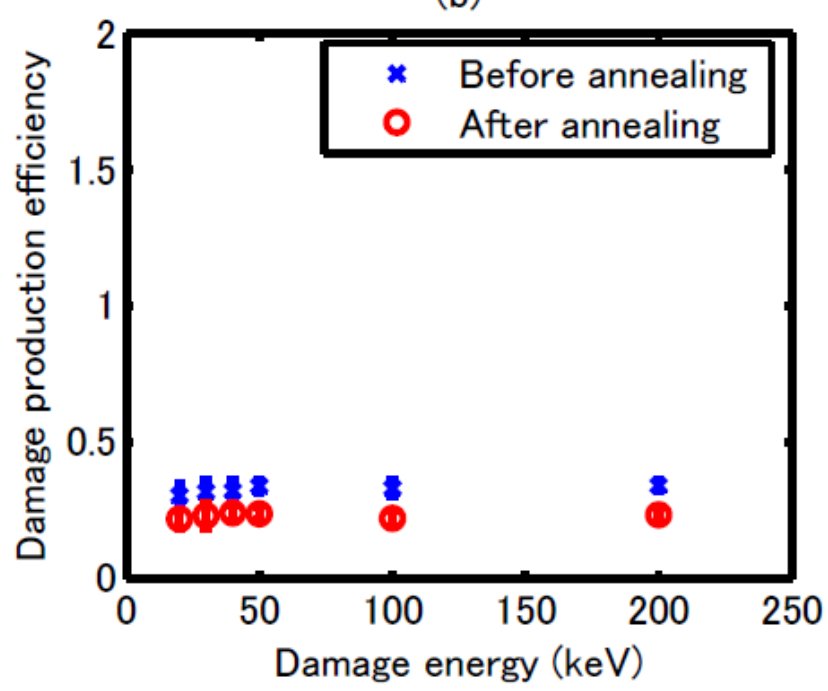

Fig. 6 Mean damage production efficiency before and after annealing
Table 4 Surviving displacements before and after annealing and the recombination ratio during annealing

\begin{tabular}{cccc}
\hline$E_{\mathrm{D}}[\mathrm{keV}]$ & $N_{\mathrm{b}} / N_{\mathrm{NRT}}$ & $N_{\mathrm{a}} / N_{\mathrm{NRT}}$ & $\left(N_{\mathrm{b}}-N_{\mathrm{a}}\right) / N_{\mathrm{b}}$ \\
\hline 0.1 & 1.45 & 1.00 & 0.31 \\
0.2 & 1.02 & 0.76 & 0.26 \\
0.5 & 0.76 & 0.63 & 0.17 \\
1.0 & 0.61 & 0.47 & 0.23 \\
2.0 & 0.51 & 0.31 & 0.38 \\
5.0 & 0.44 & 0.33 & 0.26 \\
10 & 0.34 & 0.25 & 0.27 \\
20 & 0.31 & 0.22 & 0.28 \\
30 & 0.32 & 0.23 & 0.27 \\
40 & 0.32 & 0.24 & 0.25 \\
50 & 0.34 & 0.24 & 0.30 \\
100 & 0.33 & 0.22 & 0.33 \\
200 & 0.34 & 0.23 & 0.31 \\
\hline
\end{tabular}

Similar data analyses were conducted for the other damage energy cases listed in Table 1 . The number of defects becomes less as the damage energy decreases, but the shape of graphs observed in Fig. 1 is kept for all the cases. As an example, we show the time evolution of surviving defects for $E_{\mathrm{D}}=10 \mathrm{keV}$ in Fig. 5 .

Using the annealing results given above, we examined the number of surviving displacements before and after the annealing; these values are called $N_{\mathrm{b}}$ and $N_{\mathrm{a}}$. Figure 6 shows the damage production efficiency before and after annealing, where the number of defects is normalized using the NRT displacements $\left(N_{\mathrm{NRT}}\right)$. The damage production efficiency before annealing decreases as $E_{\mathrm{D}}$ increases in the range of $E_{\mathrm{D}}<10 \mathrm{keV}$, see Fig. 6(a). In contrast, this ratio is almost independent of $E_{\mathrm{D}}$ in the range of $E_{\mathrm{D}} \geq 10 \mathrm{keV}$, see Fig. 6(b). As seen in the two figures, this characteristic feature is maintained even after annealing.

Table 4 indicates the recombination ratio during annealing, i.e. $\left(N_{\mathrm{b}}-N_{\mathrm{a}}\right) / N_{\mathrm{b}}$. For large $E_{\mathrm{D}} \mathrm{S}$, this is independent of $E_{\mathrm{D}}$ and is $\sim 0.3$. This suggests that $\sim 30 \%$ of displacements directly produced by a cascade event disappear during annealing regardless of the size of the cascade event. This indicates that the NRT displacement is proportional to surviving displacements after annealing as long as $E_{\mathrm{D}}$ is more than $\sim 10 \mathrm{keV}$ and is a meaningful damage index for long period of irradiation.

\section{Annealing Temperature Dependence}

So far, only annealing simulations at $T=300 \mathrm{~K}$ have been discussed. However, it is also important to know how the results vary with the annealing temperature. Figures $\mathbf{7}$ and $\mathbf{8}$ show the time evolution of the mean number of surviving defects for a cascade damage energy of $E_{\mathrm{D}}=100 \mathrm{keV}$ during annealing at $T=600 \mathrm{~K}$ and $T=900 \mathrm{~K}$, respectively. The obvious differences between the higher temperatures and the $300 \mathrm{~K}$ case are: (1) mobile defects start migrating and escape earlier, and (2) vacancy clusters completely disappear at the 


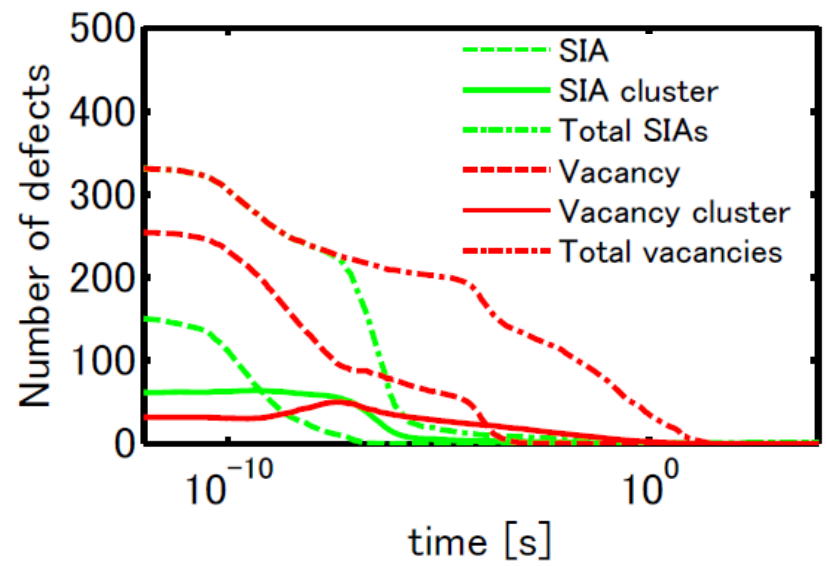

Fig. 7 Mean number of surviving defects during annealing at $600 \mathrm{~K}$ for $E_{\mathrm{D}}=100 \mathrm{keV}$

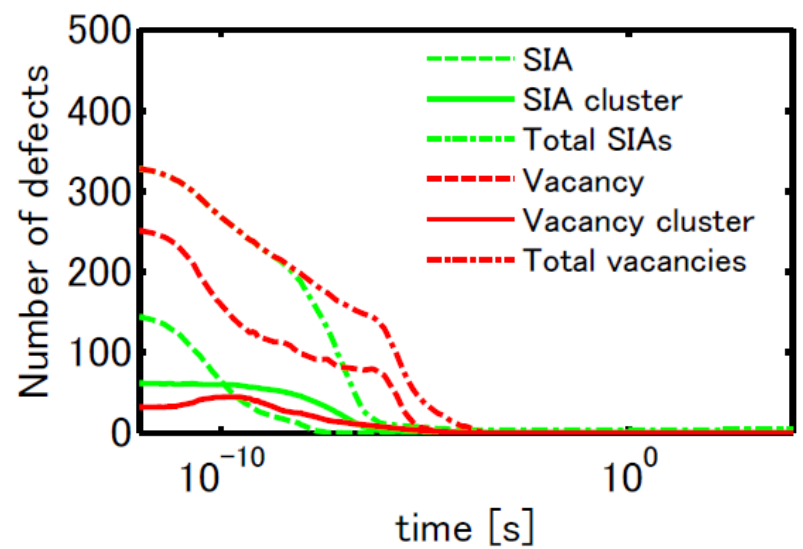

Fig. 8 Mean number of surviving defects during annealing at $900 \mathrm{~K}$ for $E_{\mathrm{D}}=100 \mathrm{keV}$

end of the simulation period because the probability of vacancy dissociation from clusters increases at high temperatures. The characteristic changes caused by high temperature are more pronounced at $900 \mathrm{~K}$.

We have also examined the change in the cluster size distribution during annealing. As shown in Figs. 7 and 8, all the vacancy clusters disappeared during annealing, thus only the size distribution of escaping SIAs were analyzed (See Fig. 9). Comparison of Fig. 4(b) with the two graphs in Fig. 9 indicates that the fraction of small SIA clusters increases as temperature increases. At higher temperatures, small SIA clusters seem to reach the boundary of the simulation box before they grow.

In Fig. 10, the temperature dependence of damage production efficiency is shown. The number of displacements after annealing decreases as the temperature increases. In particular, $N_{\mathrm{a}} / N_{\mathrm{NRT}}$ for $T=900 \mathrm{~K}$ is substantially smaller than those for $T=300 \mathrm{~K}$ and $600 \mathrm{~K}$ for high $E_{\mathrm{D}} \mathrm{s}$. This is probably because at $T=900 \mathrm{~K}$ vacancy clusters transform into single vacancies by dissociation reaction before SIAs and SIA clusters escape, and these vacancies contribute to recombination. In the previous studies of the annealing of $\mathrm{Fe},{ }^{7,8)}$ no annealing temperature dependence of this kind is reported.
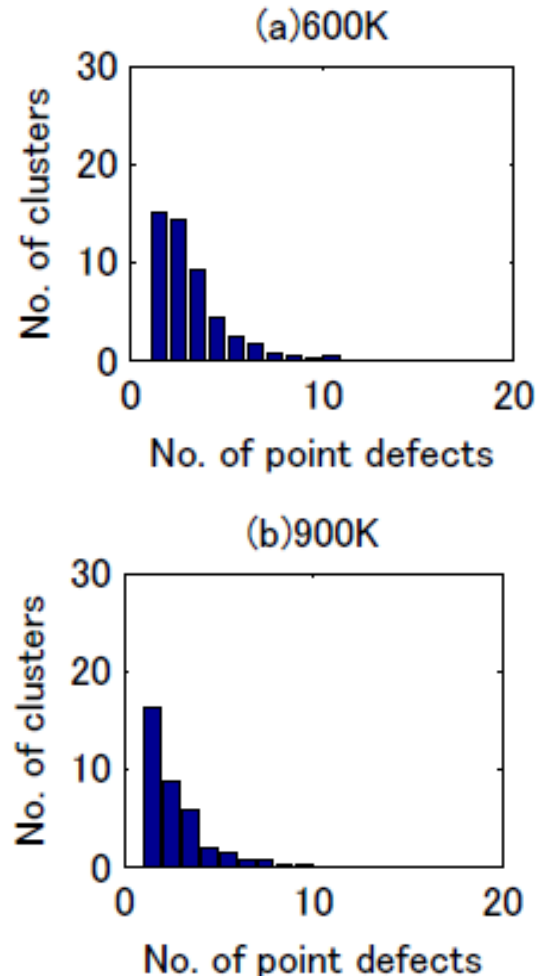

Fig. 9 Size distribution of escaped SIA clusters for $E_{\mathrm{D}}=100 \mathrm{keV}$ during annealing at $600 \mathrm{~K}$ (a) and $900 \mathrm{~K}$ (b)

\section{Influence of Dimensionality of $I_{3}$ Migration to The Defect Production Efficiency}

As mentioned in Section II. 2, the dimensionality of migration for $\mathrm{I}_{3} \mathrm{~S}$ is not yet clearly defined. Thus, we attempted to estimate the sensitivity of the annealing results to the dimensionality. This is also interesting from the standpoint of determining how much the 1D motion of small SIA loops influences the accumulation of displacement damage. We carried out the same set of annealing calculations with using $d=3$ for $\mathrm{I}_{3}$ (See Table 3 ) at $T=300 \mathrm{~K}$. Damage production efficiencies from these calculations are shown in Fig. 11 with the results shown in Fig. 6 for $d=1$ of $\mathrm{I}_{3}$. We observe that the damage production efficiency for $d=3$ is smaller. This is simply explained by the fact that the opportunity for $\mathrm{I}_{3} \mathrm{~S}$ to recombine with vacancies increases if it can migrate three-dimensionally. Despite such a small change of the input parameter, the influence was significant and was even larger than that caused by the increase in the annealing temperature to $600 \mathrm{~K}$ (See Fig. 10).

\section{Conclusion}

Using the results of molecular dynamics simulation of cascade damage, we conducted a series of annealing simulations using the kMC method. One of the characteristic quantities we focused on is damage production efficiency. Before annealing, this value is $\sim 0.3$ for PKA energies more than $\sim 10 \mathrm{keV}$, but it decreased by $\sim 30 \%$ during annealing at $300 \mathrm{~K}$ because of recombination. The recombination ratio increased as the annealing temperature increased. These results may significantly influence the long-term 
(a)

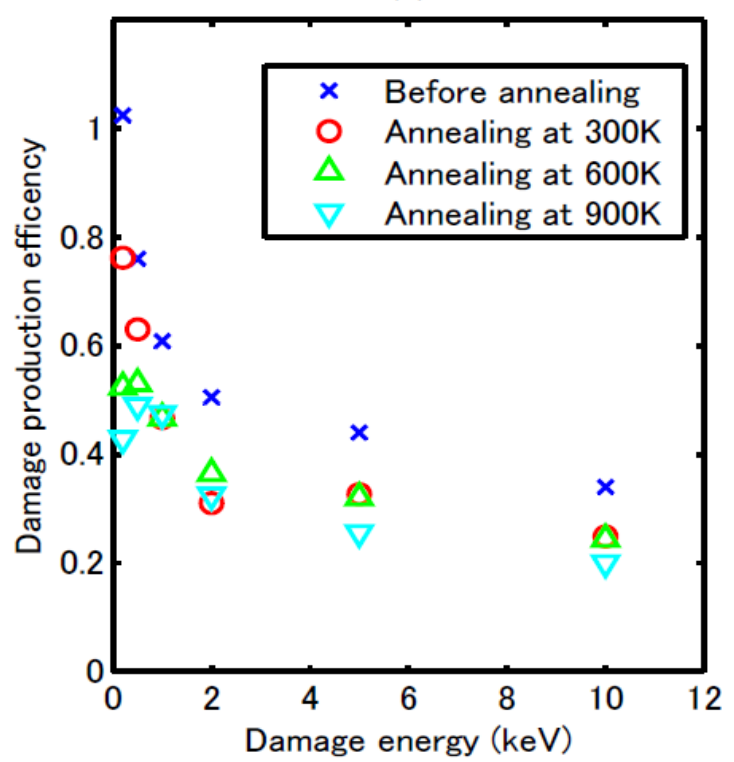

(b)

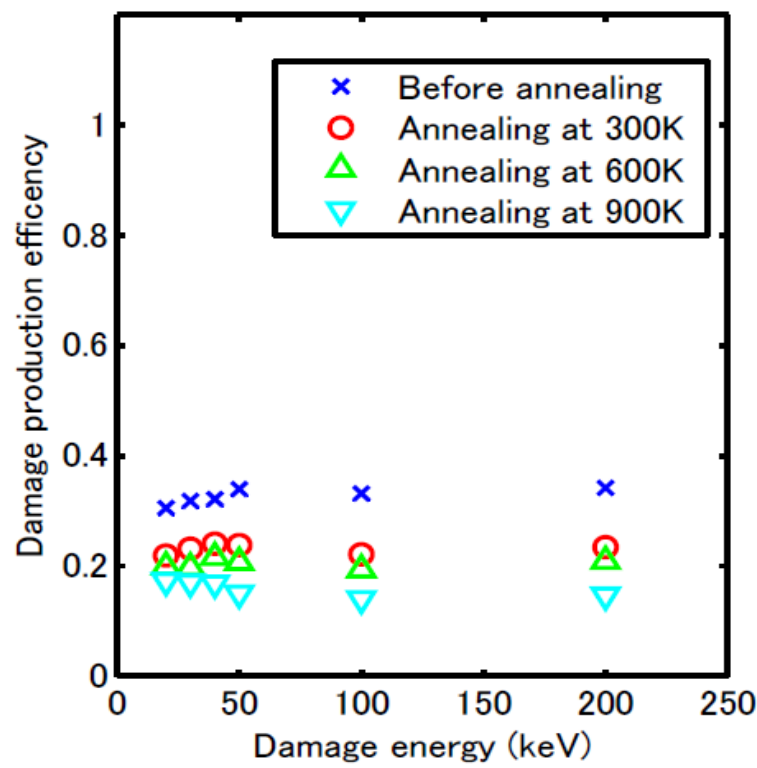

Fig. 10 Mean damage production efficiencies after annealing at various temperatures

(a)

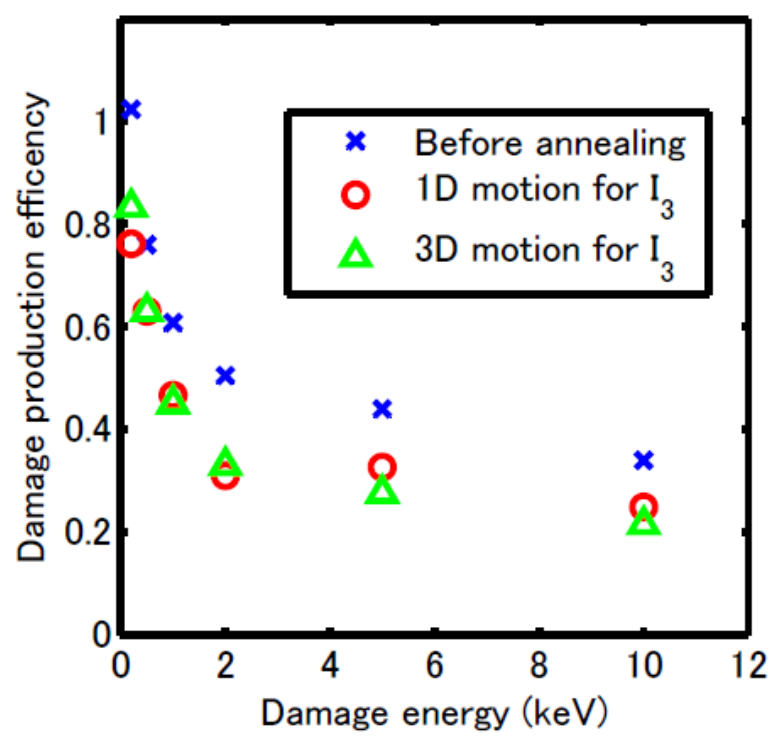

(b)

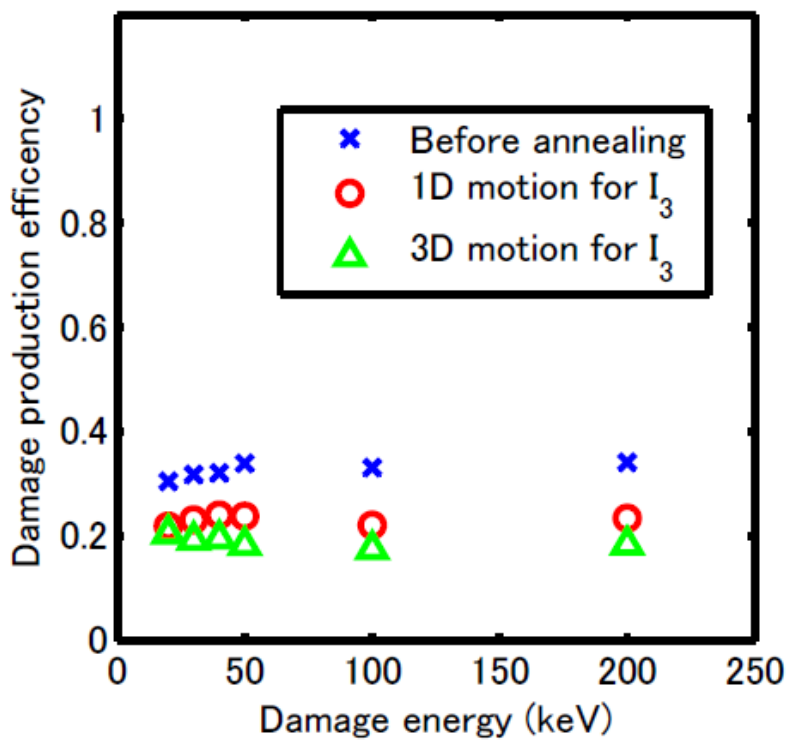

Fig. 11 Mean damage production efficiencies after annealing at various temperatures

microstructural evolution when applied to the simulation of damage accumulation in rate theory models. In addition, we quantitatively demonstrated that 1D motion of small SIA clusters can significantly influence the long-term accumulation of cascade damage.

\section{References}

1) W. J. Phythian, R. E. Stoller, A. J. E. Foreman, A. F. Calder, D. J. Bacon, J. Nucl. Mater., 223, 245-261 (1995).

2) R. E. Stoller, J. Nucl. Mater., 276, 22-32 (2000).

3) D. J. Bacon et al., J. Nucl. Mater., 323, 152-162 (2003).

4) S. I. Golubov, B. N. Singh, H. Trinkaus, J. Nucl. Mater., 276, 78-89 (2000).
5) H. L. Heinisch, B. N. Singh, J. Nucl. Mater., 232, 206-213 (1996).

6) D. G. Doran, Radiat. Eff., 2, 249-267 (1970).

7) N. Soneda, T. D. de la Rubia, Phil. Mag., A78, 995-1019 (1998).

8) F. Gao et al., Mat. Res. Soc. Symp. Proc., 540, 703-708 (1999).

9) T. S. Hudson et al., Phil. Mag., 85, 661-675 (2005).

10) C. Domain, C. S. Becquart, Phys. Rev., B65, 024103 (2002).

11) C.-C. Fu, F. Willaime, P. Ordejón, Phys. Rev. Lett., 92, 175503 (2004).

12) M. J. Norgett, M. T. Robinson, I. M. Torrens, Nucl. Eng. Des., 33, 50-54 (1975).

13) T. Suzudo el al., Mat. Res. Soc. Symp. Proc., 1215, V05-07 (2010). 
14) G. J. Ackland et al., Phil Mag., A75, 713-732 (1997).

15) G. Lucas, R. Shäublin, J. Phys. Condens. Matter., 20, 415206 (2008).

16) M. J. Caturla et al., J. Nucl. Mater., 276, 13-21 (2000).

17) C. Domain, C. S. Bequart, L. Malerba, J. Nucl. Mater., 335, 121-145 (2004).

18) A. F. Voter, in Radiation Effects in Solids, edited by K. E. Sickafus, E. A. Kotomin, and B. P. Uberuaga, Springer, NATO Publishing Unit, Dordrecht, The Netherlands, 1-24 (2006).
19) R. E. Stoller, S. I. Golubov, C. Domain, C. S. Becquart, J. Nucl. Mater., 382, 77-90 (2008).

20) F. Willaime et al., Nucl. Instr. Meth. Phys. Res., B228, 92-99 (2005).

21) D. A. Terentyev, L. Malerba, M. Hou, Phys. Rev., B75, 104108 (2007).

22) N. Anento, A. Serra, Yu. N. Osetsky, Modeling Simul. Mater. Sci. Eng., 18, 025008 (2010). 